\title{
Effect of Fabrication Parameters and Material Features on Surface Roughness of FDM Build Parts
}

\author{
Xinlong Huang ${ }^{1 *}$, Zhenhui Shen ${ }^{1}$, Shuanqiang Yang ${ }^{1}$ \\ 1.Engineering College, Fujian Jiangxia University, \\ University Town, Fuzhou, P.R.China \\ *Corresponding Author: fjjxu_huangxinlong @ fjjxu.edu.cn
}

\begin{abstract}
The part fabricated by fused deposition modeling (FDM) process is influenced by fabrication parameters viz., layer resolution, processing quality, fill mode and the processing material on surface roughness. In this work, effects of fabrication parameters are investigated experimentally. The test specimen is designed and the levels of various factor are setup respectively. Then Taguchi's parameter design is applied to find the optimum factor levels for top surface roughness and sides surface roughness. The evaluation of surface roughness is expressed as signal-to-noise $(\mathrm{S} / \mathrm{N})$ ratio . Finally the main effect plot for $\mathrm{S} / \mathrm{N}$ ratio is obtained and the optimum factor levels for top surface roughness and sides surface roughness respectively are found from analysis of experimental results.
\end{abstract}

Keywords-fused deposition modeling; surface roughness; taguchi's parameter design; main effect plot

\section{INTRODUCTION}

The rapid development of additive manufacturing (AM) resulted in the birth of a new tool in manufacturing industry and normal life due to its reduction of productive cycle [1]. This technology processed parts are fabricated from CAD model automatically without geometric restrictions. Hence, complex parts are more easily fabricated through 3D printing than traditional manufacturing technology[2]. The application technologies of 3D printing mainly include fused deposition modeling (FDM), selective laser sintering (SLS), stereo lithography apparatus (SLA), laminated object manufacturing (LOM).Among AM process, FDM which heats and extrudes melted thermoplastic filament from nozzle for building the part through the method of layer deposition until completion of part manufacturing is widely used due to its affordability and good mechanical properties[3]. However, the surface finish of part which is fabricated by FDM process is unavoidably excessively rough due to the layered production. In this regards, Several attempts and efforts have been done to solve the issue in order to improve the surface quality. The existing studies are mainly based on the analysis of modeling such as the tessellation of CAD model and the slicing procedure[4]. In addition to layered deposition method, effects of fabrication parameters related to nozzle such as the gap between nozzle and substrate and the moving speed of heating nozzle was proposed[5]. Nevertheless, Few common factors related to process parameters setting was considered correlatively.

In this research, effects of fabrication parameters and material features based on the FDM fabrication was investigated experimentally. Meanwhile, Taguchi's parameter design is applied to study effects of fabrication parameters and material features on surface roughness of FDM build parts.

\section{EXPERIMENTAL SETUPS}

The experimental parts are fabricated by the specified equipment used FDM fabrication which adopts The UP Plus 2 produced by Beijing Tier time Technology Co. Ltd. The satisfied figuration volume is $140 \times 140 \times 135 \mathrm{~mm}$ and the filament diameter is $1.75 \mathrm{~mm}$. The equipment, where the fabrication materials including ABS, ABS+, PLA are normally applied to, principally contains selectable process parameter such as processing quality, layer resolution, fill mode, sealing surface, support structure, extruder temperature, platform temperature.

A test cubic specimen of $30 \mathrm{~mm} \times 30 \mathrm{~mm} \times 35 \mathrm{~mm}$ as shown in Fig. 1 is designed for the surface roughness evaluation of FDM build parts. Due to the bottom of part is absolutely used as support generating surface, its surface quality is uncontrolled. Then top and sides of part are selected and measured only. The cubic specimen is built in 3D solid modeling software and saved as STL file afterwards.
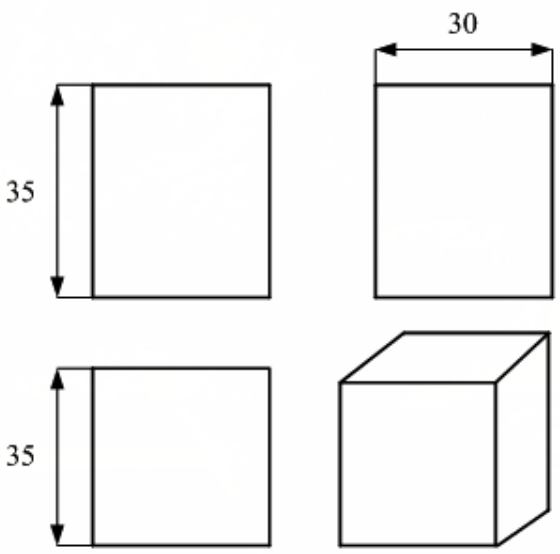

Fig.1. Detail drawing of test specimen.

As the main process parameters of the equipment ,the fabrication parameters including processing quality, layer resolution, filling method, processing material were considered as the influencing factors of the surface roughness of FDM build parts experimentally when some non-major influencing factors are excluded. The other processing parameters were set as default values during the experimental FDM printing process in addition to the adaptive extruder 
temperature adjusted by the equipment automatically. Furthermore, the surface quality of the parts is affected by the level of participating parameter. The affecting factors include layer resolution, processing quality, fill mode and the processing material are expressly represented as factors A, B,
$\mathrm{C}$ and $\mathrm{D}$ respectively. The layer resolution values 6 levels while the processing quality values 3 levels as well as fill mode. The processing material is selected from PLA, ABS, ABS+. And the specific level of each affecting factors are shown in Table I.

TABLE I.

VALUES OF ALL FACTORS

\begin{tabular}{|c|c|c|c|c|}
\hline Levels & A & B & $\mathrm{C}$ & $\mathrm{D}$ \\
\hline 1 & $0.15 \mathrm{~mm}$ & fast & Completely Filled & PLA \\
\hline 2 & $0.20 \mathrm{~mm}$ & normal & Incompletely Filled & $\mathrm{ABS}$ \\
\hline 3 & $0.25 \mathrm{~mm}$ & fine & Shell & $\mathrm{ABS}+$ \\
\hline 4 & $0.30 \mathrm{~mm}$ & & & \\
\hline 5 & $0.35 \mathrm{~mm}$ & & & \\
\hline 6 & $0.40 \mathrm{~mm}$ & & & \\
\hline
\end{tabular}

The experiment is designed based on mixed horizontal orthogonal test design (OTD) to improve the uniformity of each factor in the test and cut the test time cost consequently. To avoid influence of unknown interferences, The surface roughness measurement of specimens placed in specific areas were carried out at the normal temperature. The number of tests for top and side surface respectively in designed experiment plan is 26 equally and represented by $N$. Specifically, 4 sides of each test part were measured separately, and the average value was taken as the side roughness of each part. Measurements for Top of each test parts were operated $n$ times and $n$ is set to 6 here, and then the average surface roughness is taken as the top surface roughness of each part after the maximum and minimum values are removed. The test specimen being printed and the axes of the FMD printing equipment is shown in Fig. 2 and Fig. 3 respectively.

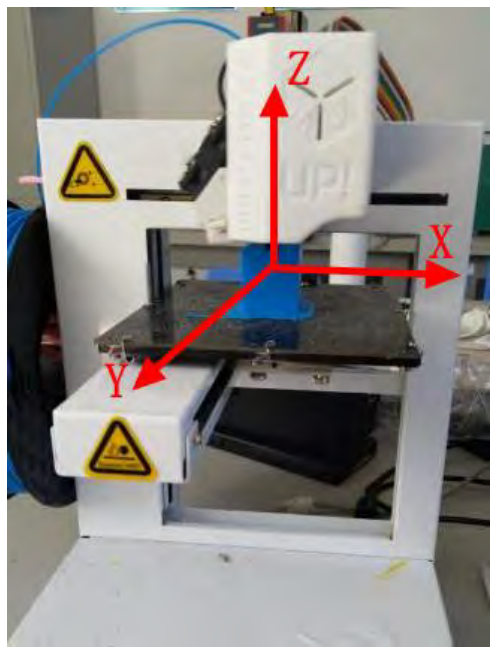

Fig.2. FDM printing of test specimen

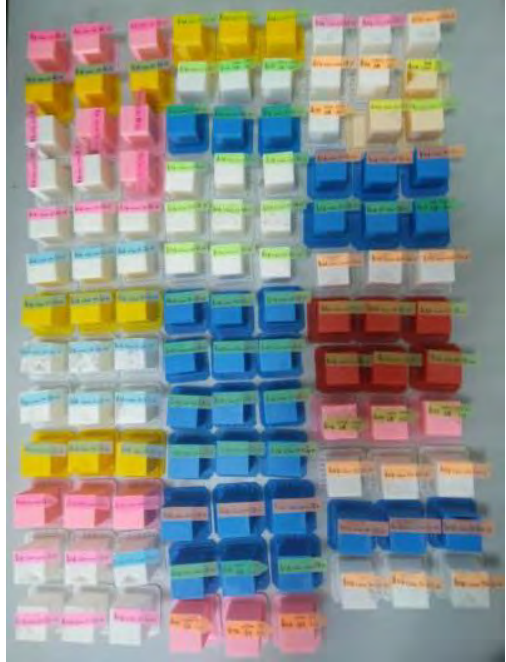

Fig.3. Test specimen

\section{RESULT ANALYSIS AND DISCUSSION}

Two readings of top surface roughness and side surface roughness were taken for each sample, and expressed by TRi and SR $i$ respectively while $i$ represents the operation index of each test and valued $1 \sim n$. The signal-to-noise ratio $(\mathrm{S} / \mathrm{N})$ is used to evaluate the influence of various factors on the total change of the result in Taguchi's parameter design. Since the objective of the experimental plan is to reduce the roughness of the top and sides, the smaller the quality characteristic is the better. $\mathrm{S} / \mathrm{N}$ denoted by $\eta$ is denoted by equation (1) and equation (2),

$$
\begin{aligned}
& \eta=-10 \log _{10}\left(\sum_{i=1}^{n} T R_{i}^{2} / n\right) \\
& \eta=-10 \log _{10}\left(\sum_{i=1}^{n} S R_{i}^{2} / n\right)
\end{aligned}
$$

The experimental data of each test surface roughness was converted into $\mathrm{S} / \mathrm{N}$ ratio as shown in Table II. The range of $\mathrm{S} / \mathrm{N}$ ratio is $[-31.54,-8.40]$ for $\mathrm{TR},[-19.00,27.77]$ for $\mathrm{SR}$. Principal effect plot for $\mathrm{S} / \mathrm{N}$ ratio used to predict optimum 
factor level with significant factors is shown in Fig.4. The results indicate that optimal factor settings for surface roughness are different between the top and the sides of the part that fabricated by FMD. According to main effect plot, the optimum factor levels for TR are A1B1C1D2 while SR are A1B1C1D3. The delta values, which indicate that the influence of the factors follows the sequence as $A>C>D>B$ for TR, $A>D>C>B$ for $S R$, stand for response of ratio is shown in Table III. Then the relative influence of each factor is determined through analysis of variance (ANOVA). Parameters of ANVOA such as degree of freedom (DOF), sum of square deviation (SS), mean square (MS), F-ratio (F) and p-value (P) is calculated and shown in Table IV and Table V. Moreover, it is considered that the factor of which the significance level is set to 0.05 has obvious influence on the surface roughness when $\mathrm{P}<0.05$. It is observed that significant factors are A,C and D for top surface roughness while A for side surface roughness.

TABLE II.

S/N RATIO OF SURFACE ROUGHNESS IN EXPERIMENT PLAN

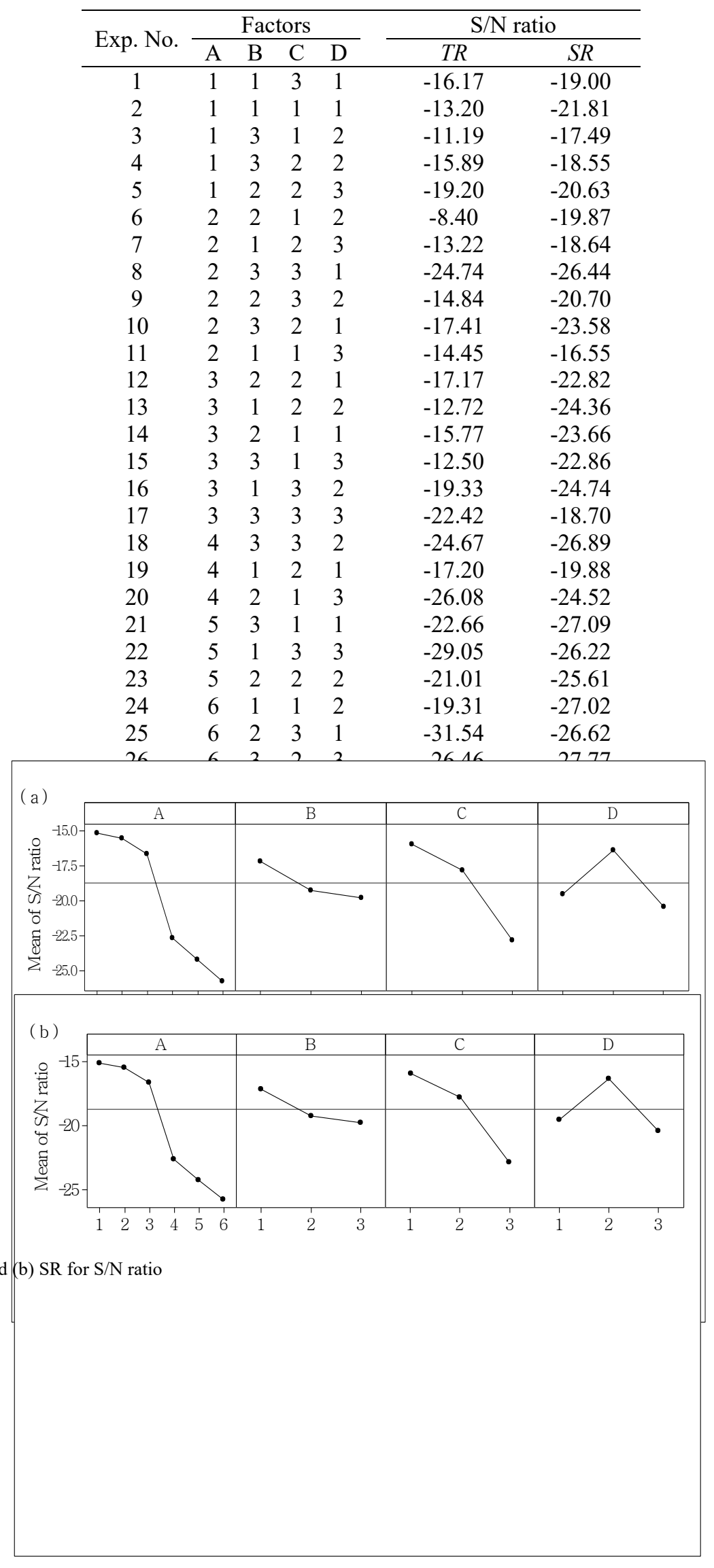


TABLE III.

RESPONSE OF S/N RATIO

\begin{tabular}{ccc}
\hline \multirow{2}{*}{ Factor } & \multicolumn{2}{c}{ Delta } \\
\cline { 2 - 3 } & $T R$ & $S R$ \\
\hline A & 10.64 & 7.64 \\
B & 2.59 & 1.24 \\
C & 6.89 & 1.35 \\
D & 4.05 & 1.45 \\
\hline
\end{tabular}

TABLE IV

ANOVA TABLE FOR S/N RATIO OF TR

\begin{tabular}{ccrrrr}
\hline Source & DOF & \multicolumn{1}{c}{ SS } & \multicolumn{1}{c}{ MS } & \multicolumn{1}{c}{ F } & \multicolumn{1}{c}{ P } \\
\hline A & 5 & 396.53 & 79.31 & 10.37 & 0 \\
B & 2 & 37.89 & 18.95 & 2.48 & 0.120 \\
C & 2 & 207.74 & 103.87 & 13.58 & 0.001 \\
D & 2 & 86.00 & 43.00 & 5.62 & 0.016 \\
Residual error & 14 & 107.06 & 7.647 & & \\
Total & 25 & 835.22 & & & \\
\hline
\end{tabular}

TABLE V.

ANOVA TABLE FOR S/N RATIO OF SR

\begin{tabular}{ccrrlc}
\hline Source & DOF & \multicolumn{1}{c}{ SS } & \multicolumn{1}{c}{ MS } & F & P \\
\hline A & 5 & 171.10 & 34.22 & 4.76 & 0.010 \\
B & 2 & 6.94 & 3.47 & 0.48 & 0.627 \\
C & 2 & 3.14 & 4.57 & 0.22 & 0.807 \\
D & 2 & 13.46 & 6.73 & 0.94 & 0.416 \\
Residual error & 14 & 100.73 & 7.20 & & \\
Total & 25 & 295.37 & & & \\
\hline
\end{tabular}

Consequently, the significant factors and levels of surface roughness vary form profile direction for FDM build parts. Factor of layer resolution influences SR and specially TR at a significance level. Factor of processing influences merely TR with a little level below 0.15 . Factor of fill mode has great influence on TR but no prominent influence on SR. Factor of processing material only has influence on TR at a normal level.

\section{CONCLUSION}

In this research, Different levels, which of four FDMbased typical processing parameters viz., layer resolution, processing quality, fill mode and the processing, influencing surface roughness were studied. Taguchi experiment design method was applied to investigate surface roughness of the FDM-based part and the optimal factors were proposed. Then the main effect plot for $\mathrm{S} / \mathrm{N}$ ratio is obtained and ANOVA is performed for $\mathrm{S} / \mathrm{N}$ ratio. The optimum factor levels for top surface roughness and sides surface roughness respectively were found from above analysis of experimental results. The main advantage of this research is the use of a efficient experimental design which popularizes assessment of significant factors for surface roughness of part fabricated in different equipments and even different process. The proposed methodology make a greater contribution to optimum fabrication parameters for FDM-based parts with complex structure and surface.

\section{ACKNOWLEDGMENTS}

This work is supported by the Education Scientific Research Project for the Young Teacher of Fujian Province, China under Grant No. JAT170613, the Natural Science Foundation of Fujian Province, China under Grant No. 2016J01727 and the Young Scientific Talent Cultivation Fund of Fujian Jiangxia University under Grant No. JXZ2015008.

\section{REFERENCES}

[1] Hlavin, Matt. 3-D Printing: The Next INDUSTRIAL REVOLUTION[J]. Appliance Design,2014,62(3).

[2] Min, K. K., Lee, I. H., \& Kim, H. C. (2018). Effect of fabrication parameters on surface roughness of FDM parts. International Journal of Precision Engineering \& Manufacturing, 19(1), 137-142. doi: 10.1007/s12541-018-0016-0

[3] R. H. Hambali, K. M. Cheong,N. Azizan. Analysis of the influence of chemical treatment to the strength and surface roughness of FDM[J]. IOP Conference Series: Materials Science and Engineering,2017,210(1).

[4] Clayton Neff,Matthew Trapuzzano,Nathan B. Crane. Impact of vapor polishing on surface quality and mechanical properties of extruded ABS[J]. Rapid Prototyping Journal,2018,24(2).

[5] Ahn D, Kweon J, Kwon S, Song J, Lee S (2009) Representation of surface roughness in fused deposition modeling. Elsevier, Journal of Materials Processing Technology, pp 5593-5600. 